\title{
Papel de los polimorfismos de los genes CFH y ARMS2 en el desarrollo de la retinopatía y la cardiopatía isquémica en la diabetes tipo 1
}

\section{Role of CFH and ARMS2 polymorphisms in retinopathy and coronary artery disease in type 1 diabetes}

\author{
M. Toni ${ }^{1}$, J. Hermida ${ }^{2}$, E. Toledo ${ }^{3}$, M.J. Goñi ${ }^{1}$, N. Díez ${ }^{4}$
}

\section{RESUMEN}

Fundamento. La diabetes tipo 1 (DT1) es un enfermedad con elevada morbilidad vascular. El objetivo de este estudio es valorar la asociación de los polimorfismos rs 1410996 del gen CFH y rs 10490924 del gen ARMS2 en pacientes con DT1.

Material y métodos. Se trata de un estudio retrospectivo, en el que se han analizado las características clínicas y los polimorfismos rs1410996 del gen CFH y rs10490924 del gen ARMS2 de 147 pacientes con DT1 valorados en la consulta de Endocrinología.

Resultados. Todos los pacientes que desarrollaron retinopatía diabética proliferativa en los primeros 20 años de evolución eran portadores del polimorfismo rs 1410996 del gen $\mathrm{CFH}$. La frecuencia del alelo de riesgo fue significativamente mayor en los pacientes con cardiopatía isquémica que en los que no la presentaban (75 frente a $53 \%, \mathrm{p}<0,001$ ).

Conclusiones. Parece existir una tendencia a aumentar el riesgo de desarrollar retinopatía diabética proliferativa en los pacientes con DT1 asociado con el polimorfismo rs 1410996 del gen CFH. Este polimorfismo parece asociarse también con el desarrollo de cardiopatía isquémica en estos pacientes. Sin embargo, el polimorfismo rs10490924 del gen ARMS2 no parece asociarse con la retinopatía ni con la cardiopatía isquémica.

Palabras clave. Diabetes tipo 1. Retinopatía diabética proliferativa. Cardiopatía isquémica. CFH. ARMS2.

\begin{abstract}
Background. Type 1 diabetes is associated with vascular morbidity. The aim of this study was to evaluate the role of polymorphisms rs $1410996 \mathrm{CFH}$ and rs 10490924 ARMS2 with proliferative diabetic retinopathy and coronary disease in type 1 diabetes patients.
\end{abstract}

Material and methods. We present a retrospective study that analyses the clinical characteristics and the polymorphisms rs1410996 CFH and rs10490924 ARMS2 of 147 type 1 diabetes patients.

Results. The patients who developed proliferative diabetic retinopathy in the first 20 years carried the rs1410996 CFH polymorphism. The overall risk-allele frequency was significantly higher among patients with coronary artery disease than in those without it (75 vs. $53 \%, \mathrm{p}<0.001)$.

Conclusions. rs $1410996 \mathrm{CFH}$ polymorphism could be associated with both proliferative diabetic retinopathy and coronary artery disease in type 1 diabetes patients. However, rs10490924 ARMS2 does not seem to be associated either with retinopathy or coronary artery disease.

Key words. Type 1 diabetes. Proliferative diabetic retinopathy. Coronary artery disease. CFH. ARMS2.

\section{Correspondencia:}

Nieves Diez Goñi

Unidad de Fisiología Humana

Facultad de Medicina. Universidad de Navarra

C/ Irunlarrea, 1

31008 Pamplona. Navarra. España

E-mail: ndiez@unav.es

\section{Becas:}

Departamento de Salud del Gobierno de Navarra. Proyecto 12075301 


\section{INTRODUCCIÓN}

La diabetes tipo 1 (DT1) es una enfermedad autoinmune ${ }^{1}$ que afecta a 10-20 millones de personas en todo el mundo ${ }^{2}$. En España la prevalencia se encuentra en torno a $0,2 \%^{3}$. En Navarra, la incidencia se sitúa en 9,5 casos nuevos por cada 100.000 habitantes/año ${ }^{4}$. Es una enfermedad crónica con complicaciones agudas y crónicas que producen disminución de la calidad y esperanza de vida.

La prevalencia de enfermedades cardiovasculares en pacientes con DT1 se encuentra entre $10,1-16,8 \%{ }^{5,6}$. Factores de riesgo de estas complicaciones son el mal control glucémico, las anomalías lipídicas, la hipertensión arterial, la obesidad y el tabaquismo. Los pacientes con DT1 tienen al menos una mortalidad diez veces mayor que los individuos de la misma edad que no padecen la enfermedad ${ }^{7}$.

La enfermedad coronaria es la causa más frecuente de mortalidad en estos pacientes $^{7,8}$. En concreto, la cardiopatía isquémica prematura causa el $27 \%$ de los fallecimientos de los pacientes de edad inferior a los 45 años ${ }^{7}$. En pacientes con DT1, la retinopatía diabética presenta una prevalencia general de un $71 \%$, y tras 20 años de evolución más del $90 \%$ presentarán dicha complicación?

La degeneración macular asociada a la edad (DMAE) neovascular, es una enfermedad degenerativa progresiva, que cursa con pérdida severa de la visión central provocada por la formación de vasos y hemorragias en la mácula. Varios estudios independientes han demostrado la asociación de dicha enfermedad con algunos polimorfismos de los genes factor $\mathrm{H}$ del complemento ( $\mathrm{CFH}$ ) y age-related maculopathy susceptibility 2 (ARMS2) ${ }^{10,11}$.

La retinopatía diabética se clasifica en proliferativa y no proliferativa, mediante valoración del fondo de ojo ${ }^{12-14}$. En la retinopatía proliferativa se desarrollan nuevos vasos sanguíneos, con riesgo importante de hemorragia y desprendimiento de retina por tracción. Por lo tanto, la re- tinopatía diabética proliferativa y la DMAE están caracterizadas por proliferación vascular donde el factor de crecimiento del endotelio vascular (VEGF) juega un papel importante. Además, ambas entidades comparten mecanismos fisiopatológicos como la generación de especies reactantes de oxígeno en respuesta a productos finales de la glicosilación avanzada, la inflamación y la activación de la vía alternativa del complemento ${ }^{15}$.

En relación a estos polimorfismos es interesante subrayar la posible asociación que pueden tener los polimorfismos del gen $\mathrm{CFH}$ en el infarto de miocardio ${ }^{16}$.

El objetivo principal de este trabajo es valorar si los polimorfismos $\mathrm{CFH}$ : rs1410996 y ARMS2: rs10490924 están asociados con el desarrollo de la retinopatía diabética proliferativa y el CFH: rs1410996, además, con el infarto de miocardio.

\section{MATERIAL Y MÉTODOS}

\section{Sujetos}

La metodología utilizada en este estudio cumple los requisitos de la Declaración de Helsinki y recibió la aprobación del Comité de Ética de Investigación de la Clínica Universidad de Navarra. El consentimiento informado fue firmado por todos los pacientes.

Se trata de una cohorte retrospectiva de 147 pacientes con DT1 reclutados en el Servicio de Endocrinología del hospital de Navarra. Se incluyeron aquellos pacientes mayores de 18 años que acudieron a revisión en el servicio de Endocrinología entre febrero de 2007 y octubre de 2009. Se recogieron diversos datos clínicos y analíticos mediante entrevista clínica y revisión de historias clínicas: sexo, fecha de nacimiento, fecha de diagnóstico de DT1, tiempo de evolución de la DT1 y control glucémico (hasta la aparición de una complicación o hasta el momento de inclusión, en los pacientes que no presentan complicaciones). Para la valoración del control glucémico, 
mediante hemoglobina glicosilada (HbA1) establecimos una escala de 0 a 2: el valor 0 equivale a $\mathrm{HbA} 1<8,9 \%$ o HbAlc (fracción más estable, la que tiene una unión con la glucosa más específica es la fracción A1c) $<7 \%$; el valor 1 equivale a $\mathrm{HbA} 1 \geq 8,9-10 \%$ o HbAlc entre 7-8\%; y finalmente, el valor 2 equivale a $\mathrm{HbA} 1>10 \%$ o $\mathrm{HbAlc}>8 \%{ }^{17}$. También se recogieron los siguientes datos: hipertensión arterial (presión arterial mayor de 130/80 mmHg), tabaquismo (número de paquetes/año), obesidad (Índice de Masa Corporal [IMC] $>30 \mathrm{~kg} / \mathrm{m}^{2}$ ), hipercolesterolemia (LDL-Colesterol $>100$ $\mathrm{mg} / \mathrm{dL}$ ), antecedentes familiares de enfermedad cardiovascular (en familiares de primer grado, edad <55 años en varones $\mathrm{y}<65$ años en mujeres). Se consideró que un paciente tenía historia de enfermedad coronaria cuando había sido diagnosticado de infarto agudo de miocardio o angina inestable, basado en los criterios clínicos, electrocardiográficos y bioquímicos habituales $^{18}$.

La presencia o severidad de la retinopatía diabética fue estudiada mediante observación oftalmoscópica del fondo de ojo, se clasificó en no proliferativa cuando se observaban microaneurismas, microexudados o microhemorragias, y en proliferativa cuando existía neovascularización retiniana o papilar, hemorragia vítrea o prerretiniana extensa, o ambas ${ }^{19}$.

\section{Detección de polimorfismos}

Se realizó la extracción de DNA de las muestras de sangre periférica de los pacientes con DT1 de acuerdo con la técnica estándar $^{20}$. Los polimorfismos rs1410996 (gen CFH) y rs10490924 (gen ARMS2) fueron determinados mediante reacción en cadena de la polimerasa (PCR) cuantitativa (TaqMan®, Applied Biosystems) siguiendo los protocolos recomendados por el fabricante.

\section{Análisis estadístico}

El estudio descriptivo de las variables cualitativas se realizó mediante test de la $\mathrm{X}^{2} \mathrm{o}$ test de Fisher exacto cuando fue necesario. Para la comparación de medias entre los grupos de participantes con y sin DT1, utilizamos el test de la t de Student, y para estudiar la correlación entre variables cuantitativas el coeficiente de correlación de Pearson. El tiempo hasta desarrollar retinopatía diabética proliferativa según el genotipo de CFH y ARMS2, se describió mediante gráficos de Kaplan-Meier y se compararon las curvas mediante el test de Breslow y el test de log rank. La valoración de la asociación entre el genotipo de CFH y ARMS2 (agrupando los sujetos heterocigotos y los homocigotos para el alelo de riesgo y definiendo como referencia los sujetos homocigotos para el alelo salvaje) con independencia de la edad del inicio de la diabetes, el sexo, hipercolesterolemia, hipertensión y el hábito tabáquico, se realizó mediante modelos de regresión de Poisson.

Así mismo, se describió el tiempo hasta desarrollar cardiopatía isquémica en pacientes diabéticos tipo 1 según el genotipo de CFH mediante gráfico de Kaplan Meier (agrupando los sujetos heterocigotos y homocigotos para el alelo de riesgo) y se empleó el test de Breslow para valorar la significación estadística entre las curvas. No se pudieron emplear modelos de regresión de Poisson ya que en la categoría de referencia (pacientes homocigotos para el alelo salvaje) no se produjo ningún evento. Para realizar los cálculos se emplearon los programas Statistical Package for Social Sciences (SPSS) versión 15.0 para Windows y STATA versión 10.

\section{RESULTADOS}

En la tabla 1 se muestran las características clínicas y las frecuencias de los polimorfismos analizados de los 147 pacientes con DT1 incluidos en el estudio. 
Tabla 1. Características clínicas y factores de riesgo vascular de todos los sujetos.

\begin{tabular}{|c|c|}
\hline & $\begin{array}{c}\text { DT1 } \\
\mathrm{n}=147\end{array}$ \\
\hline Sexo, varones, n (\%) & $85(58)$ \\
\hline Edad, años & $41 \pm 15$ \\
\hline Evolución, años & $16,0 \pm 12,2$ \\
\hline Control glucémico, escala* & $1,2 \pm 0,56$ \\
\hline Hipertensión arterial, n (\%) & $35(23,8)$ \\
\hline Dislipemia, n (\%) & $54(36,5)$ \\
\hline Hábito tabáquico, n (\%) & $73(49,8)$ \\
\hline Obesidad, n (\%) & $14(9,4)$ \\
\hline Antecedentes familiares enfermedad cardiovascular precoz, n (\%) & $10(6,9)$ \\
\hline $\begin{array}{l}\text { Macroangiopatía, n (\%) } \\
\text { Coronaria } \\
\text { Cerebral } \\
\text { Periférica }\end{array}$ & $\begin{aligned} 28 & (18,8) \\
8 & (5,8) \\
8 & (5,8) \\
18 & (12,1)\end{aligned}$ \\
\hline $\begin{array}{l}\text { Microangiopatía, n (\%) } \\
\text { Retinopatía } \\
\text { Nefropatía } \\
\text { Neuropatía }\end{array}$ & $\begin{array}{l}65(44,2) \\
57(38,7) \\
41(28,2) \\
38(26)\end{array}$ \\
\hline $\begin{array}{c}\text { Polimorfismo rs10490924 ARMS2, n (\%) } \\
\text { Homocigoto alelo salvaje (TT) } \\
\text { Heterocigoto (TC) } \\
\text { Homocigoto alelo riesgo (CC) }\end{array}$ & $\begin{array}{c}91(61,9) \\
55(37,4) \\
1(0,7)\end{array}$ \\
\hline $\begin{array}{l}\text { Polimorfismo rs1410996 CFH, n (\%) } \\
\text { Homocigoto alelo salvaje (CC) } \\
\text { Heterocigoto (CT) } \\
\text { Homocigoto alelo riesgo (TT) }\end{array}$ & $\begin{array}{c}30(20,4) \\
75(51) \\
42(28,6)\end{array}$ \\
\hline
\end{tabular}

Se representa la media \pm desviación estándar.

*Escala: 0:buen control; 1:control regular; 2: mal control

\section{Asociación de la retinopatía diabética proliferativa con los polimorfismos de los genes ARMS2 y CFH}

Los pacientes se agruparon en tres categorías diferentes según el genotipo del polimorfismo rs10490924 del gen ARMS2, definiendo el alelo $\mathrm{T}$ como el alelo de riesgo y el alelo $\mathrm{C}$ como el alelo salvaje. En los pacientes con retinopatía diabética proliferativa $(n=18)$ se estudió el tiempo libre de enfermedad y no se encontraron diferencias significativas $(\mathrm{p}=0,67)$, (Fig. 1, A). En el caso del polimorfismo rs 1410996 del gen CFH tampoco se detectaron diferencias significativas $(p=0,1)$ en el tiempo libre de en- fermedad, cuando se agruparon los pacientes según su genotipo, definiendo el alelo $\mathrm{C}$ como el alelo de riesgo y el alelo $\mathrm{T}$ como el alelo salvaje (Fig. 1, B). En los análisis de modelos de regresión de Poisson ajustados por edad de aparición de diabetes, control glucémico, sexo, hipercolesterolemia, hipertensión y hábito tabáquico, tampoco se encontró una asociación significativa de estos polimorfismos con el riesgo de padecer retinopatía diabética proliferativa [razón de tasas de incidencia $=1,53$; intervalo de confianza (IC) $95 \%(0,58-4,0)$ en el caso del gen ARMS2 y de 1,25 ; IC $95 \%(0,3-4,7)$ en el caso del gen $\mathrm{CFH}$ ]. 


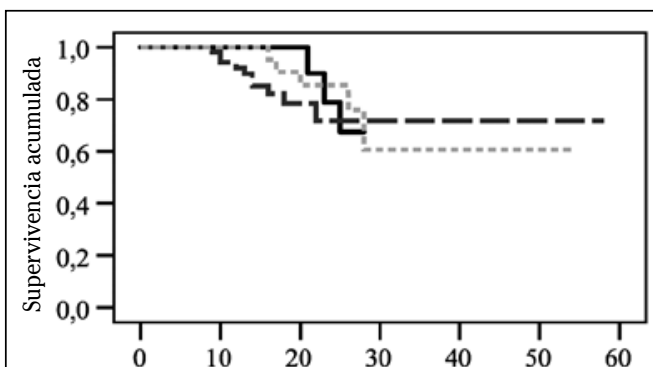

A

Tiempo de evolución en años hasta retinopatía diabética proliferativa

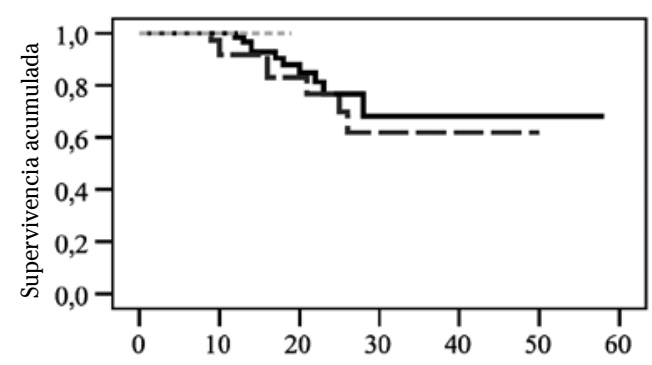

B Tiempo de evolución en años hasta retinopatía diabética proliferativa

Figura 1. Análisis del tiempo libre de retinopatía proliferativa en pacientes con DT1 de acuerdo con el genotipo del gen ARMS2 (panel A). El análisis se ha realizado con curvas de Kaplan-Meier que se compararon mediante el test $\log \operatorname{rank}\left(\chi^{2}=0,78, \mathrm{p}=0,67\right)$. Línea continúa negra, homocigoto para el alelo salvaje (CC), línea discontinua gris, heterocigoto (CT), línea punteada gris, homocigoto para el alelo de riesgo (TT). De acuerdo con el genotipo del gen CFH (panel B). El análisis se ha realizado con curvas de Kaplan-Meier que se compararon mediante el test de Breslow $\left(\chi^{2}=4,6, p=0,1\right)$. Línea continua negra, homocigoto para el alelo salvaje (TT), línea discontinua gris, heterocigoto (TC), línea punteada gris, homocigoto para el alelo de riesgo (CC).

Los resultados no demuestran un efecto, al menos importante, de estos polimorfismos sobre el riesgo de padecer retinopatía diabética proliferativa. Sin embargo, es interesante señalar que todos los pacientes que desarrollaron retinopatía proliferativa en los primeros 20 años de evolución, eran portadores del alelo $\mathrm{C}$ del polimorfismo del gen $\mathrm{CFH}$.

\section{Asociación de la cardiopatía isquémica con el polimorfismo del gen $\mathrm{CFH}$}

De los 147 pacientes en los que se han estudiado los polimorfismos del gen $\mathrm{CFH}, 8$ pacientes presentaban cardiopatía isquémica (2 de ellos padecieron dos episodios). Se calcularon las curvas de Kaplan-Meier que representan el tiempo que tardó en aparecer la cardiopatía isquémica en relación con el polimorfismo rs1410996 del gen CFH, agrupando los sujetos heterocigotos y homocigotos para el alelo de riesgo $\mathrm{C}$ en una categoría (Fig. 2). No existió una asociación significativa $(\mathrm{p}=0,29)$ entre el tiempo de aparición de la cardiopatía y el polimorfismo del gen $\mathrm{CFH}$. No fue posible emplear modelos de regresión de Poisson ya que en la categoría de referencia (pacientes homocigotos para el alelo salvaje) no se había producido ningún evento.

La frecuencia del alelo de riesgo $\mathrm{C}$ en los pacientes con cardiopatía isquémica fue significativamente más alta que en los que no la padecieron (75 frente a $53 \%, \mathrm{p}<0,001$ ).

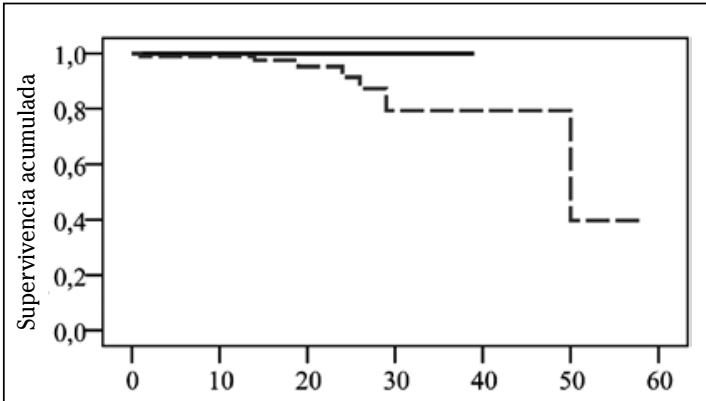

Tiempo de evolución en años hasta infarto de miocardio
Figura 2. Análisis del tiempo libre de cardiopatía isquémica en pacientes con DT1 de acuerdo con el genotipo del gen $\mathrm{CFH}$. El análisis se ha realizado con curvas de Kaplan-Meier que se compararon mediante el test de Breslow $\left(\chi^{2}=1,07\right.$, $\mathrm{p}=0,29$ ). Línea continua negra, homocigoto para el alelo salvaje (TT), línea discontinua gris (portadores del alelo de riesgo C (TC y CC). 


\section{DISCUSIÓN}

La DT1 es una enfermedad con elevada morbilidad que aumenta sustancialmente el riesgo de enfermedad coronaria y ceguera en quienes la padecen. Resulta esencial identificar los posibles factores involucrados en el desarrollo de estas complicaciones. En nuestro estudio no hemos podido demostrar una asociación clara entre los polimorfismos rs10490924 del gen ARMS2 y rs1410996 del gen CFH con la retinopatía proliferativa o la cardiopatía en los pacientes con DT1.

Sin embargo, los resultados del estudio sugieren un discreto aumento del riesgo de desarrollo de retinopatía diabética proliferativa en los pacientes portadores del alelo de riesgo C del polimorfismo rs1410996 del gen CFH, aunque sin llegar a la significación estadística. Es interesante señalar que todos los pacientes que desarrollaron retinopatía proliferativa en los primeros veinte años de evolución de la diabetes eran portadores del alelo $\mathrm{C}$ del polimorfismo rs1410996 del gen CFH. Este polimorfismo se asocia con el riesgo de degeneración macular asociada a la edad ${ }^{10}$, una enfermedad caracterizada por la angiogénesis patológica de la coroides que conduce a la ceguera. La retinopatía diabética proliferativa es un proceso donde se produce angiogénesis en la retina. Se ha demostrado que la inflamación y consiguiente activación del complemento a través de la vía alternativa están involucrados en el desarrollo de ambas entidades. El aumento de la permeabilidad y la consiguiente proliferación vascular mediadas por el VEGF son fenómenos comunes a las dos patologías ${ }^{21,22}$. Este es el primer estudio en el que se analiza el riesgo de padecer retinopatía diabética proliferativa en pacientes con DT1 asociado con estos polimorfismos, aunque existe un estudio en pacientes con diabetes tipo 2 , en el que no se encuentra asociación ${ }^{15}$.

Recientemente, se han publicado algunos trabajos analizando la asociación entre el polimorfismo rs1410996 del gen CFH y la cardiopatía isquémica. En un estudio prospectivo realizado en una población general mayor de 55 años se encontró una asociación importante entre el estado homocigoto y el riesgo de infarto de miocardio ${ }^{16}$. Esta asociación aumentaba significativamente con la presencia de otros factores de riesgo cardiovascular como el tabaco, la hipercolesterolemia y la diabetes tipo 2. En otros estudios este resultado no se ha podido confirmar $^{23}$ e incluso se han publicado resultados en los que se ha hallado una asociación inversa ${ }^{24}$. Una posible explicación para esta discrepancia es la necesidad de la presencia de otros factores de riesgo cardiovascular para que se manifieste el efecto deletéreo del polimorfismo rs1410996 del gen $\mathrm{CFH}$. Por este motivo hemos estudiado su efecto en una población con DT1 que es un factor de riesgo cardiovascular importante y no estudiado anteriormente. Hemos encontrado diferencias significativas en la frecuencia alélica del polimorfismo del gen $\mathrm{CFH}$ y una tendencia que parece indicar un aumento de riesgo de padecer cardiopatía isquémica. Si se confirman estos hallazgos, la detección de este polimorfismo podría ser útil para personalizar las estrategias terapéuticas de prevención de cardiopatía en pacientes con DT1.

Este estudio tiene limitaciones que se deben tener en cuenta para valorar correctamente los resultados. Como es un estudio retrospectivo, se han perdido pacientes que han fallecido durante la evolución de su enfermedad por patologías que podrían estar relacionadas con los polimorfismos analizados. Estas pérdidas pueden influir en el resultado, fundamentalmente reduciendo el efecto de estos polimorfismos sobre la retinopatía y la cardiopatía. Otra limitación del estudio es el número relativamente pequeño de pacientes incluidos y la dispersión del tiempo de evolución de los mismos, lo que hace que el porcentaje de retinopatía sea menor del esperado.

De este estudio podemos concluir que parece existir una ligera tendencia a aumentar el riesgo de desarrollo de retinopatía diabética proliferativa en los pacientes con DT1 asociado con la presencia del alelo C del polimorfismo rs1410996 del gen CFH. Así mismo, se observa una tendencia a aumentar el riesgo de desarrollo de cardiopatía isquémica en los pacientes con DT1 asociado con el alelo C del polimorfismo rs1410996 del gen CFH. 


\section{Agradecimientos}

Agradecemos a los endocrinólogos del Complejo Hospitalario de Navarra por facilitarnos el reclutamiento de pacientes. A Maider Esparza, y a todo el personal del laboratorio de Trombosis y Hemostasia del CIMA por su ayuda en la recogida, procesamiento y determinación de polimorfismos de las muestras.

\section{BIBLIOGRAFÍA}

1. George S. Eisenbarth. Diabetes mellitus tipo 1. En: Kahn CR, Weir GC, King GL, Jacobson AL, Moses AC, Smith RJ, editors. Joslin's Diabetes Mellitus, Massachusets: Boston, 2007: 399-424.

2. Expert Committee on the Diagnosis and Classification of Diabetes Mellitus. American Diabetes Association, Alexandria, Virginia, USA. Report of expert committee on the diagnosis and classification of diabetes mellitus. Diabetes Care 2003; 26 (Suppl.1): S5-S20.

3. Goday A, Caltell C, Tresserras JL, Lloveras G. La diabetes mellitus tipo 1 en España. Estimación de la incidencia anual y su distribución por Comunidades Autónomas y provincias. Endocrinología 1994; 41: 301-304.

4. Ruiz-Ramos M, Escolar-Pujolar A, MayoralSánchez E, Corral-San Laureano F, FernándezFERNÁNDEZ I. Mellitus diabetes in Spain: death rates, prevalence, impact, costs and inequalities. Gac Sanit 2006; 20 (Suppl. 1): 15-24.

5. Sibal L, Law HN, Gebbie J, Dashora UK, Agarwal SC, Home P. Predicting the development of macrovascular disease in people with type 1 diabetes: A 9-year follow-up study. Ann N Y Acad Sci 2006; 1084: 191-207.

6. Cusick M, Meleth AD, Agron E, Fisher MR, ReEd GF, KNATterud GL et al. Associations of mortality and diabetes complications in patients with type 1 and type 2 diabetes: early treatment diabetic retinopathy study report no. 27. Diabetes Care 2005; 28: 617-625.

7. The effect of intensive treatment of diabetes on the development and progression of longterm complications in insulin-dependent diabetes mellitus. The Diabetes Control and Complications Trial Research Group. N Engl J Med 1993; 329: 977-986.

8. Orchard TJ, Costacou T, Kretowski A, Nesto RW. Type 1 diabetes and coronary artery disease. Diabetes Care 2006; 29: 2528-2538.
9. The diabetes control and complications trial/epidemiology of diabetes interventions and complications research Group. Retinopathy and nephropathy in patients with type 1 diabetes four years after a trial of intensive therapy. N Engl J Med 2000; 342: 381-389.

10. Fritsche LG, Loenhardt T, Janssen A, Fisher SA, Rivera A, KeIlhauer CN et al. Age-related macular degeneration is associated with an unstable ARMS2 (LOC387715) mRNA. Nat Genet 2008; 40: 892-896.

11. Maller J, George S, Purcell S, Fagerness J, AltsHULER D, DALY MJ et al. Common variation in three genes, including a noncoding variant in $\mathrm{CFH}$, strongly influences risk of age-related macular degeneration. Nat Genet 2006; 38: 1055-1059.

12. Andonegui J, Serrano L, Egúzkiza A. eOftalmología: estado actual y tendencias futuras. An Sist Sanit Navar 2010; 33: 79-91.

13. ForGA L. Retinografía no midriática para la detección de la retinopatía diabética. Un ejemplo de adaptación al medio. An Sist Sanit Navar 2010; 33: 255-258.

14. Gibelalde A, Ruiz-Miguel M, Mendicute J, Ayerdi S, Martínez-Zabalegi D. Prevalencia de retinopatía diabética mediante cribado con retinógrafo no midriático. An Sist Sanit Navar 2010; 33: $271-276$.

15. Doney AS, Leese GP, Olson J, Morris AD, Palmer $\mathrm{CN}$. The $\mathrm{Y} 402 \mathrm{H}$ variant of complement factor $\mathrm{H}$ is associated with age-related macular degeneration but not with diabetic retinal disease in the Go-DARTS study. Diabet Med 2009; 26 : 460-465.

16. Kardys I, Klaver CC, Despriet DD, Bergen AA, UitTERlinden AG, HoFman A et al. A common polymorphism in the complement factor $\mathrm{H}$ gene is associated with increased risk of myocardial infarction: the Rotterdam Study. J Am Coll Cardiol 2006; 47: 1568-1575.

17. Koshy A, Giacherio D, Hayward R. Hemoglobin A1c Fact Sheet.U-M MDRTC Chemistry Laboratory.

18. Grupo de Trabajo para el manejo del síndrome coronario agudo (SCA) en pacientes sin elevación persistente del segmento ST de la Sociedad Europea de Cardiología (ESC). Autores/Miembros del Grupo de Trabajo: Christian W. Hamm (Moderador) (Alemania), Jean-Pierre Bassand(Co-Moderador), (Francia), Stefan Agewall (Noruega), Jeroen Bax (Paises Bajos), Eric Boersma (Paises Bajos), Hector Bueno (Espana), Pio Caso (Italia), Dariusz Dudek (Polonia), Stephan Gielen (Alemania), Kurt Huber (Austria), Magnus Ohman (Estados Unidos), 
Mark C. Petrie (Reino Unido),Frank Sonntag (Alemania), Miguel Sousa Uva (Portugal), Robert F. Storey (Reino Unido), William Wijns (Belgica) y Doron Zahger (Israel). Guía de practica clínica de la ESC para el manejo del síndrome coronario agudo en pacientes sin elevación persistente del segmento ST. Rev Esp Cardiol. 2012; 65: 173. e1-e55.

19. Aliseda D, Berastegui L. Retinopatía diabética. An Sist Sanit Navar 2008; 31 (Supl. 3): 23-34.

20. Montes R, Ruiz de Gaona E, Martínez-González MA, Alberca I, Hermida J. The c.-1639G > A polymorphism of the VKORC1 gene is a major determinant of the response to acenocoumarol in anticoagulated patients. Br J Haematol 2006; 133: 183-187.

21. Gerl VB, Bohl J, Pitz S, Stoffelns B, Pfeiffer N, BHAKDI S. Extensive deposits of complement C3d and C5b-9 in the choriocapillaries of eyes of patients with diabetic retinopathy. Invest Ophthalmol Vis Sci 2002; 43: 11041108.

22. Zhang J, Gerhardinger C, Lorenzi M. Early complement activation and decreased levels of glycosylphosphatidylinositol-anchored complement inhibitors in human and experimental diabetic retinopathy. Diabetes 2002; 51: 3499-3504.

23. Nicaud V, Francomme C, Ruidavets JB, Luc G, ARVEILER D, KEE F, et al. Lack of association between complement factor $\mathrm{H}$ polymorphism and coronary artery disease or myocardial infarction. J Mol Med 2007; 85: 771-775.

24. Pai JK, Manson JE, Rexrode KM, Albert CM, Hunter DJ, Rimm EB. Complement factor $\mathrm{H}$ $(\mathrm{Y} 402 \mathrm{H})$ polymorphism and risk of coronary heart disease in US men and women. Eur Heart J 2007; 28: 1297-1303. 